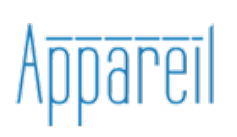

Appareil

18 | 2017

Art et médium 2 : les média dans l'art

\title{
Sur les intermédia
}

\section{Dick Higgins}

Traducteur : Pascal Krajewski

\section{OpenEdition \\ Journals}

Édition électronique

URL : http://journals.openedition.org/appareil/2379

DOI : 10.4000/appareil.2379

ISSN : 2101-0714

Éditeur

MSH Paris Nord

\section{Référence électronique}

Dick Higgins, « Sur les intermédia », Appareil [En ligne], 18 | 2017, mis en ligne le 19 septembre 2017 consulté le 30 juillet 2020. URL : http://journals.openedition.org/appareil/2379 ; DOI : https://doi.org/ 10.4000/appareil.2379

Ce document a été généré automatiquement le 30 juillet 2020.

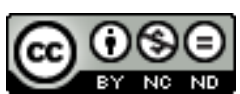

Appareil est mis à disposition selon les termes de la Licence Creative Commons Attribution - Pas d'Utilisation Commerciale - Pas de Modification 4.0 International. 


\title{
Sur les intermédia
}

\author{
Dick Higgins \\ Traduction : Pascal Krajewski
}

\section{NOTE DE L'AUTEUR}

Traduction de l'anglais (États-Unis) par Pascal Krajewski avec l'aimable autorisation du Dick Higgins Estate. Le texte original est sous copyright du Dick Higgins Estate.

Le présent essai regroupe 3 textes : "Intermedia”, qui fut publié en février 1966 dans Something Else Newsletter ( $\mathrm{n}^{\circ}$ 1, New York, Something Else Press) ; "Statement on intermedia" fut écrit le 3 août 1966 et publié en juillet 1967 dans Dé-coll/age* 6, (Wolf Vostell [ed], New York, Something Else Press). "Synesthesia and Intersenses: Intermedia" est un article double, reprenant l'article "Intermedia" de 1966, et lui ajoutant une suite rédigée en 1981. Il fut publié dans Dick Higgins, Horizons: The Poetics and Theory of the Intermedia, Chicago, Southern Illinois University Press, 1984.

\section{Intermédia (1966)}

1 La plupart des œuvres les plus intéressantes produites actuellement semblent se situer à l'intersection de plusieurs médiums ${ }^{1}$. Ce n'est pas fortuit. L'idée de cloisonner les médiums naquit à la Renaissance. L'opinion selon laquelle un tableau est fait de peinture apposée sur une toile, ou celle qui stipule qu'une sculpture ne devrait pas être peinte, semble caractéristique d'un certain genre de pensée sociale - celle qui catégorise et divise la société en noblesse (et ses subdivisions variées), petite noblesse sans titre, artisans, serfs et travailleurs sans terre - que nous appellerons la conception féodale de la grande chaîne des êtres. Cette approche essentiellement mécaniste continua d'être pertinente tout au long des deux premières révolutions industrielles (dont nous sortons tout juste) et elle l'est encore à l'heure actuelle de l'automatisation, qui constitue de fait une troisième révolution industrielle. 
Cependant, contrairement aux problèmes politiques, les problèmes sociaux qui sont les nôtres aujourd'hui n'autorisent plus cette approche cloisonnante. Nous abordons à l'aube d'une société sans classe, pour laquelle toute séparation en catégories rigides s'avérera totalement inadaptée. Ce mouvement ne concerne pas plus l'Est que l'Ouest (et vice versa). Castro travaille dans des champs de canne à sucre. Pendant la grève des métros, Lindsay, le maire de New York, dut se rendre à pied au travail. Les millionnaires déjeunent à Horn et Hardart's $\mathrm{s}^{2}$. Ce type de populisme révèle une tendance grandissante et non pas déclinante.

3 Pour en faire l'expérience, il suffit de regarder l'art qui se dit appartenir - avec quelle rigidité gratuite! - à telle ou telle forme. Nous voyons des peintures. Que sont-elles après tout ? Des objets, chers, faits main, destinés à orner les murs des gens riches, ou bien, profitant de leur munificence (ou de celle du gouvernement), à être partagés avec le plus grand nombre, à qui ils promettent d'insuffler un sentiment de grandeur. Mais elles n'offrent aucune possibilité de dialogue.

Le pop art ? Comment pourrait-il jouer un rôle dans l'art à venir ? Il est fade. Il est pur. Il emploie des éléments de la vie quotidienne sans commentaire, et ainsi, en acceptant silencieusement la misère de cette vie et son aridité, il les dédouane. D'ailleurs, le pop et l'op'art sont tous deux déjà morts parce qu'ils se contentent, en utilisant leur médium, d'activer les anciennes fonctions de l'art - la décoration et l'évocation de la grandeur -, sans tenir compte du contenu précis des intentions des artistes. Aucune des ingénieuses théories du monstrueux sieur Ivan Geldoway ${ }^{3}$ ne les exempte de leur colossal ennui et de leur absence de pertinence. Milord tient sa galerie dans Folle Avenue et y expose sa marchandise. Il est protégé par une poignée de laquais balourds bien utiles qui semblent croire que la Vie sera toujours ainsi. Répondant au doigt et à l'œil, voici encore Monsieur Insensible Irritable, un grand prêtre modérément bien informé, qui apparemment méprise la Flamme qu'il devrait veiller, pour préférer ce qui le titille. Quoi qu'il en soit, Milord a besoin de ses services, parce que, lui, pauvre petite chose, n'a pas le temps ni l'énergie « d'en être » au-delà de son nom et de ses dollars; s'informer, découvrir ce qui se passe: voilà qui est tout simplement trooooooooop fatigant. Ainsi, bien protégé et bien conseillé, il se balade benoîtement dans nos rues dans un pur style Louis XIV.

5 Or, cette scène n'est pas uniquement l'apanage du monde institutionnel de la peinture. Elle est absolument naturelle (et intrinsèque) au concept de médium pur, qu'il s'agisse de tableaux ou de quelque autre objet précieux. C'est ainsi que ces objets sont marketés, puisqu'ainsi va le monde auquel ils appartiennent et ont partie liée. Et pourtant, cette idée de "L'État, c'est moi » sera bientôt remplacée par celle d'un «Après moi, le déluge ", et d'ailleurs, si le Monde de l'Art était mieux informé, il se rendrait compte que le déluge a déjà commencé.

6 À quand remontent ses débuts? Nul besoin pour nous de suivre le fil de l'histoire dans tous ses détails. Pour une part, si les objets de Duchamp continuent de nous fasciner tandis que la voix de Picasso s'affadit, c'est parce que les œuvres de Duchamp sont véritablement entre les média (entre la sculpture et quelque chose d'autre), tandis qu'un Picasso trouvera sa place convenable parmi les ornements peints. De la même façon, en pénétrant un domaine situé entre le collage et la photographie, l'Allemand John Heartfield a produit sans doute les meilleures images de notre siècle et sûrement l'art politique le plus puissant qui n'ait jamais été fait. 
7 Le ready made, ou objet trouvé, et donc aussi en un sens « intermédium » puisqu'il n'a pas été conçu pour se conformer à un médium pur, suggère généralement cette idée, et donc désigne une certaine zone dans le domaine qui s'étend entre la sphère générale des médiums artistiques et celle des média du quotidien. Cependant, à l'heure actuelle, les zones de ce type sont relativement peu explorées, par rapport à celles qui s'étendent entre les médiums des différents arts. Par exemple, je ne connais pas de travaux qui auraient volontairement été placés dans l'intermédium entre la peinture et les chaussures. La chose qui s'en approche le plus est peut-être la sculpture de Claes Oldenburg, située quelque part entre la sculpture et le hamburger ou la glace esquimau, bien que rien de tout cela ne soit la source de ces images elles-mêmes. Il y a encore fort à faire dans cette direction pour pouvoir mettre à jour des possibilités esthétiquement encourageantes.

8 Au milieu des années 1950, nombreux furent les peintres qui commencèrent à comprendre l'absurdité fondamentale de l'expressionnisme abstrait, mode dominante de l'époque. Des peintres tels Allan Kaprow et Robert Rauschenberg aux États-Unis ou Wolf Vostell en Allemagne se tournèrent vers le collage (ou pour ce dernier, vers le décollage), pratique consistant à faire des œuvres en ajoutant ou en enlevant, en remplaçant et substituant ou en modifiant les composants d'un travail visuel. Ils commencèrent à intégrer des objets de plus en plus incongrus dans leur travail. Rauschenberg appela ses constructions des "combines», et alla jusqu'à exposer sur l'une d'elle un bouc empaillé - éclaboussé de peinture et portant un pneu en caoutchouc autour du $\mathrm{cou}^{4}$. Kaprow, plus philosophe et inquiet, médita sur la relation entre le spectateur et l'œuvre. Il intégra des miroirs à ses objets, pour que le spectateur puisse s'y sentir englobé. Comme ce n'était pas assez physique, il fit des collages enveloppants qui entouraient le spectateur; et il les appela des «environnements». Finalement, à l'automne 1958, il commença à intégrer des gens en chair et en os dans ses collages, et il appela cela un " happening».

9 Le théâtre avec une avant-scène est un rejeton des idéaux de l'ordre social du XVII siècle. Pourtant, et c'est fort remarquable, il n'y a qu'une infime différence structurelle entre les drames de Davenant et ceux d'Edward Albee ${ }^{5}$, qui ne présente en tout cas rien de comparable avec les différences que l'on trouve dans la construction de pompes hydrauliques ou dans les moyens de transport de masse. Tout se passe comme si les implications sociales et technologiques des deux premières révolutions industrielles avaient été complètement éludées. Une pièce de théâtre est toujours divisée selon la même mécanique : il y a les acteurs, les gens de la production, un public distinct et un script explicite. Une fois lancé, semblable au monstre de Frankenstein, le cours des choses est immuable, comme maudit par son incapacité à refléter ce qui l'entoure. $\mathrm{Vu}$ notre mentalité populiste actuelle, il est difficile d'attacher de l'importance à ce théâtre traditionnel - au-delà du respect qu'on nous a appris à lui témoigner. C'est tout juste si ses innovations mineures nourrissent nos conversations à la sortie des spectacles: la scène était ronde et non pas carrée, l'autre jour la scène tournait sur elle-même, ici la pièce était plutôt absurde et fantasque (après tout, Pinter est notre J. M. Barrie moderne - à moins que cet honneur ne revienne plutôt à Beckett). Chaque année, de moins en moins de personnes se déplacent dans les théâtres privés de Broadway. Les spectacles y sont de plus en plus stupides, reflets de l'estime que les producteurs portent à notre mentalité (ou bien est-ce la leur qui ainsi se révèle ?). Le meilleur du théâtre classique ne se trouve même plus sur Broadway, il faut se rendre à quelques 
kilomètres de là, à l'église du Mémorial Judson. Pourtant, nos écoles de théâtre continuent de déverser des milliers de professionnels du spectacle sur les milliers déjà en place, pour des emplois qui n'existeront tout simplement plus dans vingt ans. Faut-il blâmer les syndicats? Ou les loyers et les taxes foncières? Bien sûr que non. Les productions subventionnées, sponsorisées par les musées comme le Lincoln Center à New York, n'amènent pas un nouveau public, pas plus qu'elles n'en rameutent un ancien, car le médium de ces pièces semble déplacé et artificiel dans notre nouveau milieu social. Nous avons besoin de plus de portabilité et de flexibilité, et voilà précisément ce que ne peut fournir le théâtre traditionnel. Il fut conçu pour Versailles et ses nobliaux sédentaires, pas pour les démons turbulents et motorisés qui font 1000 bornes par semaine. Versailles ne nous est plus guère audible, à nous autres qui pensons à $100 \mathrm{~km} /$ h.

À l'opposé, certains (dont moi), partant de l'idée même de théâtre, ont déclaré la guerre au script, simple ramassis d'événements séquentiels. L'improvisation ne me satisfaisait pas non plus, car les acteurs se contentaient de jouer en imitant un script. Ainsi commençais-je à travailler comme si le temps et le séquençage pouvaient être totalement suspendus, non pas en les ignorant ce qui eût été simplement illogique, mais en transformant systématiquement ces éléments structurels en paramètres changeants. Et l'arrêt des changements sonnait la fin de mes pièces. En 1958, j'écrivis une pièce, Stacked Deck, dans laquelle n'importe quel événement pouvait survenir à tout moment, pour peu qu'il soit déclenché par un signal ${ }^{6}$. Les signaux étaient produits par des lumières colorées. Comme les lumières colorées pouvaient être allumées à tous les endroits où on les avait disposées, et comme les réactions du public produisaient aussi des signaux déclencheurs de situations, la séparation acteur-public se dissolvait et une situation de happening s'établissait, même si l'utilisation qui était faite de l'environnement et de l'imagerie n'avait pas ici d'objectif d'abord visuel. Au même moment, issu du domaine des expérimentations sur la notation graphique, Al Hansen nous rejoignit, puis ce fut au tour Nam June Paik et Benjamin Patterson (tous deux alors en Allemagne), qui venaient d'un domaine musical élargi dans lequel les événements musicaux spécifiques étaient fréquemment remplacés par des actions non-musicales.

11 Ainsi le happening se développa-t-il comme un intermédium, une terre vierge qui se tenait entre le collage, la musique et le théâtre. Il n'est régi par aucune règle ; chaque œuvre détermine ses propres médium et forme, en fonction de ses besoins. Le concept lui-même se comprend mieux par ce qu'il n'est pas, plutôt que par ce qu'il est. C'est en l'approchant que nous sommes de nouveau pionniers, et nous le resterons aussi longtemps qu'il y aura de grandes marges de manœuvre et aucun voisin alentour dans un rayon de plusieurs kilomètres. Bien sûr, un tel concept est très perturbant pour ceux dont la mentalité est compartimentée. Le Time, le magazine Life et les Grands Prêtres ont régulièrement annoncé la mort des happenings depuis que cette forme a pris de l'importance à la fin des années 1950. Mais tout cela dit davantage de la qualité de leur information que de la vitalité de cette forme.

12 Nous avons relevé des intermédia dans le théâtre ainsi que dans les arts visuels, les happenings et certains types de constructions physiques. Pour des questions de place, nous ne pourrons continuer le recensement des intermédia qui germent entre les autres sphères. Cependant, j'aimerais suggérer que l'utilisation des intermédia est plus ou moins universelle dans tous les beaux-arts; en effet, la continuité, bien plus que la catégorisation, est la véritable marque de fabrique de notre nouvelle mentalité. On peut 
trouver des équivalents au happening en musique, par exemple dans le travail de compositeurs tels Philip Corner et John Cage, qui explorent les intermédia entre la musique et la philosophie, ou avec Joe Jones dont les instruments musicaux automates se logent dans l'intermédium entre la musique et la sculpture. Les poèmes construits d'Emmett Williams et de Robert Filliou constituent certainement un intermédium entre la poésie et la sculpture. Est-il possible de parler du recours aux intermédia comme d'un large mouvement inclusif dont Dada, le futurisme et le surréalisme auraient été des phases précoces, précédant l'énorme lame de fond qui nous frappe aujourd'hui ? Ou est-il plus raisonnable de considérer l'utilisation des intermédia comme une innovation historique irréversible, davantage comparable au développement, par exemple, de la musique instrumentale, qu'à celui, disons, du romantisme?

\section{Déclaration sur les intermédia (1966)}

13 L'art est l'un des moyens par lesquels les gens communiquent. Je peux difficilement imaginer une personne qui s'attaquerait sérieusement à un quelconque moyen de communication en tant que tel. Nos véritables ennemis sont ceux qui nous envoient mourir dans des guerres inutiles ou vivre des vies réduites au labeur - et non pas les gens qui utilisent d'autres moyens de communication que ceux que nous jugeons les plus appropriés à la situation présente. S'attaquer à ceux-ci revient simplement à produire une diversion dont seuls nos véritables ennemis tirent parti.

14 Cependant, du fait de l'accélération de l'alphabétisation de masse, et à cause de la télévision et de la radio, nos sensibilités ont changé. La complexité même de ce bouleversement nous redonne le goût de la simplicité et d'un art fondé sur les images sous-jacentes, éternel outil de l'artiste cherchant à se faire entendre. Comme nous le fîmes avec les cubistes, nous en appelons à une nouvelle façon de regarder les choses, qui serait plus totale, plus immédiate, parce que nous sommes plus impatients et plus soucieux d'aller aux images primitives. Ainsi s'explique l'impact des happenings, des pièces-événements (events), des films en mixed media. Nous ne réclamons plus de discours enflammés nous enjoignant à prendre les armes contre un océan de problèmes, nous voulons seulement que cela soit fait. L'art qui s'y attelle le plus directement est celui qui permet cette immédiateté, avec un minimum de distractions.

Dieu seul sait à quel point les moyens, les goûts et les visions psychédéliques vont venir accélérer le processus. À mon avis, cela ne changera pas tout, mais cela contribuera à intensifier une tendance déjà présente.

Depuis une dizaine d'années environ, les artistes changent leur médium pour s'adapter à cette situation, à tel point que les médiums dans leurs formes traditionnelles se sont disloqués et sont devenus de simples points de repère pour les puristes. Telle une combustion spontanée prenant dans le monde entier, l'idée a germé selon laquelle ces balises sont arbitraires et qu'elles ne sont utiles que comme outils indispensables pour déclarer que telle ou telle œuvre est fondamentalement de la musique, tout en étant aussi de la poésie. Telle est l'approche intermédiale : elle souligne la dialectique entre les médiums. Un compositeur est un homme mort, sauf s'il compose pour tous les médiums et pour son monde.

17 Par conséquent, ne paraitt-il pas raisonnable qu'ayant découvert le régime des intermédia (ce qui devait peut-être n'arriver qu'en les approchant par des moyens formels et même abstraits), le problème central soit à présent non plus seulement le 
nouveau problème formel consistant à apprendre à les utiliser, mais le nouveau problème social s'interrogeant sur la finalité de leur utilisation. Ayant découvert des outils à l'impact immédiat, à quoi allons-nous les employer? Si, contrairement à McLuhan et d'autres qui ont les premiers éclairé le problème, nous supposons qu'il y a des forces dangereuses à la manœuvre dans notre monde, ne paraît-il pas approprié de nous allier contre elles, et en guise de nouveau sujet de travail, de nous tourner vers ce qui nous tient vraiment à cœur et ce que nous aimons ou détestons? Se pourrait-il que le problème central des dix prochaines années, pour tous les artistes, dans toutes les formes possibles, soit moins la découverte à venir de nouveaux médiums et intermédia, que la découverte d'une nouvelle façon de nous servir de ce qui nous importe vraiment, d'une manière qui soit à la fois appropriée et explicite ? Le vieil adage ne fut jamais plus vrai qu'aujourd'hui : il ne suffit pas de dire qu'une chose est comme cela, pour qu'elle soit vraiment ainsi. Il ne suffit pas de parler du Vietnam ou de la crise dans nos mouvements syndicalistes, pour nous garantir contre toute stérilité. Nous devons trouver les moyens de dire ce qui doit être dit, au vu de nos nouveaux modes de communication. Pour cela, nous aurons besoin de nouvelles tribunes, organisations, critères, sources d'information. Il y a du pain sur la planche, peut-être comme jamais. Et nous devons à présent nous atteler à la tâche.

\section{Synesthésie et Intersens : les intermédia (1981)}

En 1965, quand j'ai écrit les mots ci-dessus ${ }^{7}$, il s'agissait simplement pour moi de proposer un biais qui permettrait d'entrer dans certaines œuvres déjà existantes, dont les formes avaient un caractère si inhabituel que de nombreux spectateurs, auditeurs ou lecteurs potentiels étaient "déboutés » par elles. À cette époque, le monde était plein de poèmes concrets, de happenings, de poésie sonore, d'environnements, et d'autres développements plus ou moins neufs. Faute de pouvoir regarder dans l'œuvre, en la forçant à se tenir tranquille un instant et à se laisser catégoriserer, il était fort probable que le public la congédie comme relevant de l'«avant-garde: pour seuls spécialistes ». Pour tout amateur non-spécialiste, cela pouvait être frustrant - à l'époque, on voulait pouvoir bien connaître l'art de son époque, parce qu'on voulait percevoir sa propre voix ou sa propre personne dans l'œuvre, sans avoir à recourir à l'Histoire ou à des jugements historiques. Tel était bien l'art dont les horizons répondraient aux nôtres.

Le véhicule que je choisis, le mot "intermédia ", apparaît dans les écrits de 1812 de Samuel Taylor Coleridge ${ }^{8}$ exactement dans le sens qu'il a aujourd'hui - définissant les œuvres qui tombent conceptuellement entre les médiums déjà reconnus - et j'avais pour ma part déjà commencé à employer ce terme quelques années avant d'écrire mon petit essai, dans des conférences ou au détour de conversations. De plus, dans le cadre de mon projet visant à populariser ce qui était connu comme «d'avant-garde : pour seuls spécialistes ", c'est-à-dire en quelque sorte à démythifier cet art, j'étais devenu l'éditeur d'une petite maison d'édition, Something Else Press (1964-1974), qui publiait de nombreuses sources de première main et de nombreux matériaux au sujet de ces arts nouveaux (tout comme elle réinterrogeait des œuvres du passé qui nous semblaient mériter une attention renouvelée - tels que les travaux de Gertrude Stein, des dadaïstes, du compositeur Henri Cowell, etc.). Il semblait tout à fait idiot de publier mon court essai dans un magazine déjà existant, où il aurait sans doute été rangé et 
oublié. Il fut donc publié dans le premier bulletin de la Something Else Newsletter, et envoyé à nos clients, à tous nos contacts, à ceux à qui je pressentais qu'une telle idée pourrait être utile (par exemple, à des artistes faisant ce qui me semblait être des œuvres intermédiales, et aux critiques qui pourraient être aptes à en discuter). À tout prendre, $j$ 'ai distribué près de 10000 exemplaires de cet essai, soit autant que je pouvais me le permettre; et j'ai encouragé sa republication par quiconque m'en demandait l'autorisation. Il fut republié sept ou huit fois, pour autant que je sache, et il vit encore comme chapitre de différents livres, pas seulement les miens, mais aussi dans des anthologies, aux côtés d'autres textes de l'époque, ou dans des compendiums récapitulatifs.

Le terme mena rapidement sa vie propre, comme je l'espérais. Il n'était en aucun cas ma propriété. Il fut repris, usé et abusé, souvent confondu avec l'expression «mixed media ». Ce dernier est un mot vénérable de la critique d'art, qui caractérise les œuvres produites grâce à plus d'un médium, comme la peinture à l'huile et la gouache. Mais, par extension, il peut aussi s'adapter à des formes telles que l'opéra, pour lequel la musique, le livret et la mise en scène sont aisément distinguables : jamais le spectateur d'opéra n'a de doute sur le fait qu'il est en train de voir la mise en scène, le spectacle sur les planches, ou bien d'écouter la musique, etc. Beaucoup de belles œuvres ont été faites en mixed media : par exemple, des peintures incorporant des poèmes dans leurs champs visuels. Mais ici, on peut toujours distinguer ce qui est quoi.

D'un autre côté, avec les intermédia, l'élément visuel (la peinture) se fond conceptuellement avec les mots. On peut avoir de la calligraphe abstraite, de la poésie concrète, ou de la "poésie visuelle " (pas n'importe quel poème avec un fort élément visuel, mais des œuvres visuelles dans lesquelles apparaît un poème, souvent sous forme de photographie, ou bien dans lesquelles le matériel visuel photographié est présenté comme une séquence avec sa grammaire propre, comme si chaque élément visuel était le mot d'une phrase, comme on en trouve chez Jean-François Bory ou Duane Michaels).

Encore une fois, le terme n'est pas prescriptif; il ne cherche pas à s'imposer ni à se présenter comme modèle à suivre pour faire des œuvres grandes ou nouvelles. Il dit simplement que des œuvres intermédiales existent. Se méprendre sur ce sujet conduirait à une autre erreur : penser à tort que les intermédia sont nécessairement, par nature, datés dans le temps; qu'ils sont quelque chose d'enraciné dans les années 1960, une sorte de mouvement artistique caractéristique d'une époque. Il n'y a jamais eu aucun mouvement intermédial, il ne pouvait pas y en avoir. L'intermédialité a toujours été une possibilité depuis les temps les plus reculés, et bien que certains commissaires bien-intentionnés tiennent à la disqualifier comme formaliste et par conséquent antipopulaire, elle revient comme possibilité chaque fois que resurgit le désir de fusionner deux médiums préexistants (ou plus). On peut faire semblant de ne pas la voir; on peut être comme Rosalind Krauss, critique très respectée, qui déclara lors d'une conférence à Iowa City en 1981: "Je me consacre à l'idée d'essayer d'enterrer l'avant-garde », et faire ce qu'elle fit en l'attaquant, en l'ignorant, elle et ses implications, et, ce qui est bien pire, en présentant la théorie comme une fin en soi, de sorte que toute œuvre d'art devenait, au mieux, un appendice superflu à la théorie. Mais il y a toujours une avant-garde, dans le sens où quelqu'un quelque part est toujours en train d'essayer de faire quelque chose qui va ajouter des possibilités pour tout le monde, et où ce « tout le monde » finira par suivre largement ce " quelqu'un » et 
par utiliser, dans son travail artisanal routinier, toutes les innovations offertes. «L'avant-garde » est juste une métaphore conventionnelle, issue du champ militaire (et du milieu du XIX ${ }^{e}$ siècle), pour qui une avant-garde est ce qui se déplace en avance du gros des troupes. "L'avant-garde » est relative, pas absolue. Un poète conservateur peut être au moins moralement avant-gardiste s'il tend vers toujours plus de pureté et d'intégrité, s'il vise à l'intensif, la métaphore ou la perfection de la rime. D'autres essayeront de suivre, même quand ils n'en ont pas les moyens; et c'est pourquoi cette métaphore conserve sa pertinence.

Mais quand on pense à l'avant-garde des formes et des médiums, on pense bien souvent à des artistes qui, pour diverses raisons, interrogent ces formes et ces médiums. Ils peuvent en rejeter certains (par exemple, les prédécesseurs d'André Breton dans le mouvement français Dada rejetèrent le roman et ils appartenaient bien à l'avantgarde ; puis quand André Breton choisit de se tourner vers un certain type de roman possible, cela provoqua une fracture entre son groupe, les futurs surréalistes, et l'ancien - or, ce nouveau groupe constituait lui aussi l'avant-garde). Ils peuvent en créer d'autres. Et souvent cette création de nouveaux médiums s'accomplit par la fusion d'anciens ; c'était assez banal à la fin des années 1950 et au début des années 1960, avec les fusions formelles que j'ai déjà mentionnées. Aucune œuvre n'a jamais été bonne à cause de son intermédialité 9 . L'intermédialité était simplement une partie de la manière d'être de l'œuvre ; la reconnaître rendait le classement de l'œuvre plus aisé, de sorte que les gens pouvaient comprendre l'œuvre et ses significations.

En outre, les intermédia ont tendance à devenir des médiums familiers. Le roman visuel est devenu une forme très reconnaissable pour nous. Nous en avons connu de nombreux ces vingt dernières années. Il est redevenu incongru de souligner son ancien statut intermédial (situé entre art visuel et texte); on veut aujourd'hui savoir de quoi parle tel ou tel roman visuel et comment il marche ; quant à la notion d'intermédialité, elle est devenue superflue. Il en va de même avec la poésie visuelle et la poésie sonore. Dans les arts de la performance, il y eut d'abord le happening, qui était proche des " événements " (events); certains artistes de ce mouvement se lancèrent dans Fluxus, et d'autre pas. Au moins un artiste Fluxus, Alison Knowles, évolua tellement dans son travail qu'elle finit par se retrouver en train de faire ce que d'autres nouveaux artistes - dont beaucoup faisaient grand cas de distinguer leur travail des happenings, des événements et de Fluxus - appelaient soit "art performance ", soit "performance art ». Où pourrions-nous trouver un principe commun à tout ceci? Dans l'intermédialité qui les caractérise : tous, ils relèvent du même intermédium, une fusion conceptuelle d'un scénario, d'une visualité et, assez souvent, d'éléments sonores. Mais l'intermédialité explique-t-elle l'unicité ou la valeur du meilleur de cet art performance (ou performance art)? Je ne pense pas. Certaines œuvres vont rester et serviront de modèle pour définir leur genre, d'autres tomberont dans l'oubli. Au mieux, nous avions besoin de la notion d'intermédialité pour suggérer leur trajectoire historique, pour montrer leur pedigree parfois caché (comme on pourrait le faire pour les happenings, en remontant leur lignée jusqu'à Dada et les manifestations futuristes). Mais si une œuvre doit un jour devenir importante pour un grand nombre de gens, ce sera parce que son nouveau médium lui octroie une large signifiance, et pas seulement parce que sa nature formelle lui assure une pertinence.

Et donc, il faut comprendre ces quelques lignes comme une prescription à l'usage des gens désireux d'user du terme «intermédia»: certes, il permet d'entrer dans une 
œuvre qui autrement semblerait opaque et impénétrable, mais une fois que cette percée a été faite, il n'est plus utile d'insister sur l'intermédialité d'une œuvre. Aucun artiste reconnu ne pourrait rester longtemps un «artiste intermédial» l'intermédialité se ferait encombrante : elle lesterait l'artiste soucieux de répondre aux injonctions de l'œuvre à accomplir et désireux de tracer les horizons d'une ère nouvelle, à destination des générations futures d'auditeurs, de lecteurs, d'observateurs, et qui répondront à leurs propres horizons. Ce qui fut utile au commencement, deviendrait, en s'installant, une obsession qui freinerait la circulation de l'œuvre, et aussi ses besoins et ses potentialités. On regrette souvent l'adhérence d'un artiste à un petit groupe de dogmes : «l'artiste d'un mouvement » en est un parfait exemple - il ou elle continue d'adhérer aux enseignements de son mouvement, bien longtemps après que ce dernier a perdu tout intérêt. Il y a le vieux "futuriste", le vieil «abstractionniste abstrait», le vieil «artiste pop». Être vieux, en ce sens, c'est en quelque sorte créer un genre d'académisme, bon pour fournir des exemples à une classe d'élèves ( Okay tout le monde, cette semaine, je veux que chacun d'entre vous fasse une peinture pop art »), peut-être utile à des fins heuristiques, mais reste peu à même d'ouvrir de nouveaux horizons pour l'artiste ou ses observateurs, ses écouteurs, ses lecteurs.

Et voilà comment je quitterai ce sujet des intermédia. C'est aujourd'hui encore, comme ça l'était en 1965, un moyen utile pour approcher certaines œuvres; on se demande «Entre quelles choses connues cette œuvre se tient-elle?». Mais cette démarche est bien plus utile au début d'un processus critique qu'à ses étapes ultérieures. Peut-être n'ai-je pas vu cela à l'époque, mais cela m'apparaît aujourd'hui clairement. Peut-être que dans toute l'excitation de ce qui était alors pour moi une découverte, je l'ai surestimée. Je ne souhaite pas compenser cela par une seconde erreur de jugement, en la sous-évaluant aujourd'hui. Cependant, il me semble que si l'on veut améliorer sa compréhension d'une œuvre donnée, il faut regarder ailleurs - vers tous les aspects d'une œuvre (et pas seulement vers ses origines formelles), ainsi que vers les horizons que l'œuvre implique - afin de trouver un procédé herméneutique approprié pour voir le tout de l'œuvre dans la relation qu'elle entretient avec moi.

\section{NOTES}

1. Higgins n'emploie que les mots «medium» au singulier et «media » au pluriel. Nous avons préféré le pluriel « médiums » quand il référait spécifiquement à la sphère de l'art. [NdT]

2. Célèbre chaîne de restaurants où la nourriture était fournie par distribution automatique. $[\mathrm{NdT}]$

3. Tout ce passage se transforme en une satire ad hominem qu'il est difficile de rendre. "M. Ivan Geldoway» est une sorte de chimère diabolique, fusionnant Ivan Karp (galeriste), Henry Geldzahler (commissaire) et Lawrence Alloway (critique). Higgins en parle comme d'un « combine ", référence aux combine paintings de Rauschenberg, œuvre hybride entre sculpture, assemblage et peinture. [NdT]

4. Robert Rauschenberg, Monogram, 1955-59. Il s'agit d'un Freestanding combine. [NdT] 
5. Deux dramaturges anglophones séparés par trois siècles: Sir William Davenant, Angleterre (1606-1668) et Edward Albee, États-Unis (1928-2016). [NdT]

6. Pièce remontée le 28 février 2015 au Savage Vanguard Theater d'Austin au Texas. Captation visible sur Youtube : https://www.youtube.com/watch?v=DB2wVNmA-5k [NdT]

7. Ceux du texte I : « Intermédia [1966]». [NdT]

8. Le mot « intermedium » apparaît en effet dans la troisième leçon, « Sur Spenser », de Coleridge.

Il désigne plutôt un point logé entre deux figures de rhétorique, et se rapproche du mot « intermédiaire ». "L'allégorie narrative se distingue de la mythologie comme la réalité du symbole; elle est, pour le dire d'une formule, l'intermedium exact entre la personne et la personnification ». Samuel Taylor Coleridge, « Lecture III [3/2/1818]: On Spenser », dans A Course of lectures [1818], dans Henry Nelson Coleridge (ed.), The literary remains of Samuel Taylor Coleridge, W. Pickering, 1836, en ligne: http://onlinebooks.library.upenn.edu/webbin/gutbook/lookup? num $=8488$. [NdT]

9. En 1965, j'aurais peut-être soutenu l'inverse. Mais des recherches sur l'histoire des poésies visuelle et sonore m'ont suggéré que virtuellement toutes les fusions possibles ont été faites, ou au moins étaient préfigurées, à une époque ou à une autre, et que l'émergence réelle d'une certaine partie seulement de ces fusions a plus à voir avec l'économie ou les modes d'une époque qu'avec son véritable Zeitgeist.

\section{RÉSUMÉS}

I. Dans "Intermedia", Higgins introduit deux substantifs : intermedium et son pluriel intermedia. L'auteur commence par noter les changements sociaux frappant son époque, qui passe d'une société divisée, de castes, à une société égalitaire sans classe. À son sens, l'irruption, dans la sphère de l'art, d'œuvres inclassables, venant se loger entre les médiums établis, participe du même esprit. Il appelle ces œuvres hybrides des «intermédia ». Elles peuvent être à la croisée de différents médiums artistiques, ou même au croisement des médiums artistiques et de nos média mondains. Elles constituent à ses yeux ce que l'art de son époque produit de plus intéressant.

Ainsi, en peinture. Quoi qu'en disent leurs thuriféraires, le pop'art ou l'expressionnisme abstrait, parce qu'ils conservent la vieille logique de séparation des médiums, ne sont plus pertinents. Higgins leur préférera Fluxus, le collage, les combine paintings de Rauschenberg, ou les happenings de Kaprow (Duchamp est à cet égard précurseur).

Dans le domaine de la scène, Higgins ne perçoit de réelles nouveautés grosses d'avenir que dans les inventions tournant autour des happenings et de la participation du public.

Selon lui, l'intermédium ne révèle pas un mouvement artistique, datable, mais une tendance majeure et constante de l'art, qui se manifeste dans les années 1950-1960 avec une acuité sans pareille.

II. On trouve dans "Statement on intermedia" l'adjectif intermedial. Higgins tient, dans ce texte, un discours politiquement engagé. Constatant les enjeux politiques nouveaux de son temps, il voit dans l'utilisation de l'art, et singulièrement des intermédia, le moyen pour les artistes de participer à une lutte politique. L'intermédium est alors la seule façon de renouer directement avec la vie, de faire vaciller les anciennes catégories, et de produire un art engagé. "Telle est l'approche intermédiale: elle souligne la dialectique entre les médiums ». Le défi alors se dédouble : pour l'œuvre, la question ne concerne plus sa forme, mais son discours ; pour l'artiste, l'objectif n'est plus l'exploration de sa technique, mais l'usage politique de son art. 
III. Le substantif intermediality apparaît dans "Synesthesia and Intersenses: Intermedia". Quinze ans après l'écriture de l'essai introduisant le terme "intermédium », le théoricien revient sur cette notion et le contexte de son apparition. Il s'agissait alors pour lui de forger un concept utile à l'amateur pour comprendre et apprécier son art d'avant-garde, un art nécessaire à toute la sphère de l'art. Présenté dans sa revue Something Else Press, le terme « intermédium » se répandit et même échappa en partie à son créateur. Higgins précise en quoi il continue de distinguer les intermedia des mixed media: ceux-ci agencent des médiums côte à côte quand ceux-là les fusionnent. Autre précision importante: le terme d'intermédium n'est pas prescriptif, mais descriptif. Higgins avoue ici l'évolution de sa pensée, en ramenant à sa juste valeur la notion qu'il proposa : elle est pertinente pour décrire et entrer dans un certain type d'œuvres, elle n'est pas valable pour juger de la qualité intrinsèque d'une œuvre. Enfin, l'auteur insiste sur le caractère non historique de l'art intermédial, qui est en fait un mouvement présent dans l'art de toutes les époques ( l'intermédialité a toujours été une possibilité ») et dont les œuvres perdent leur caractère d'avant-garde avec le temps.

La notion d'intermédialité doit donc être utilisée puis délaissée, et non pas respectée comme un dogme. Elle court dans l'histoire de l'art et parmi de nombreux arts, si différents se pensent-ils. Elle ne peut garder sa vitalité qu'en refusant de devenir un nouvel académisme. L'art, quel qu'il soit, doit d'abord et avant tout ouvrir de nouveaux horizons à son public.

\section{INDEX}

Mots-clés : intermedia, media, avant-garde, mélange, entre, happening, théâtre, fluxus 